\section{Rapid and precise read-out of terahertz sensor by high-speed asynchronous optical sampling}

\section{G. Klatt, M. Nagel, T. Dekorsy and A. Bartels}

High-speed asynchronous optical sampling is used for the rapid and precise determination of transmission resonances of terahertz surface sensors based on asymmetric double split ring resonator arrays. The sensor response represented by a characteristic resonant frequency is determined to $0.66113 \mathrm{THz}$ with an accuracy of $\pm 325 \mathrm{MHz}$ within 2 seconds of read-out time.

Introduction: Frequency selective surfaces (FSS) are based on metallic resonator arrays with tailored transmittance and reflectivity properties [1]. Recently, it has been proposed in a numerical study to use THzFSS based on asymmetric split ring resonators as a sensor for detecting biomolecular sample films with a thickness of only $10 \mathrm{~nm}$ [2]. Because large biomolecules, e.g. DNA, exhibit a multitude of inherent vibrational modes, terahertz radiation is ideal to excite and probe these modes and to detect DNA by its binding state specific terahertz properties [3]. Applying substances to an FSS changes the resonant response of the sensor array which can be monitored in transmittance or reflectivity [4-6]. In the case of asymmetric split ring resonators there is an extraordinarily strong field enhancement in the gap region of the ring resonator [7]. Compared to classic free space radiation analysis $[8,9]$ this field localisation leads to a much higher sensitivity of THz-FSS based sensing reducing the required amount of sample material significantly. For diagnostic applications, however, up to 100 arrayed gene sensors need to be read out in a reasonably short time. Up to now, sensor read-out has been done with rather slow operating conventional terahertz time-domain spectrometers based on mechanical delay stages [10]. Here we present investigations on terahertz-FSS resonant frequency read-out performed with a terahertz time-domain spectrometer based on high-speed asynchronous optical sampling (ASOPS).

Surface sensor design and fabrication: The THz-FSS consists of asymmetric double-split metallic rings arranged on a quadratic grid with a pitch of $p=197 \mu \mathrm{m}$ (see Fig. 1). The rings are placed on top of a $27 \mu \mathrm{m}$-thick polymer substrate made of benzocyclobutene (BCB) with an assumed permittivity $\varepsilon_{\mathrm{r}}=2.6$ and dielectric loss factor $\tan \delta=$ 0.0001 . The FSS is fabricated by spin-on deposition of the BCB layer on a silicon host substrate including thermal curing at $210^{\circ} \mathrm{C}$ for $2 \mathrm{~h}$. The ring structures are patterned on top of the $\mathrm{BCB}$ using a standard photolithography process, e-beam metal evaporation $(\mathrm{Cr} / \mathrm{Au}$ with $10 \mathrm{~nm} / 200 \mathrm{~nm}$ thickness) and photoresist lift-off. In a last step the FSS is lifted off the silicon substrate in $\mathrm{KOH}$ solution.

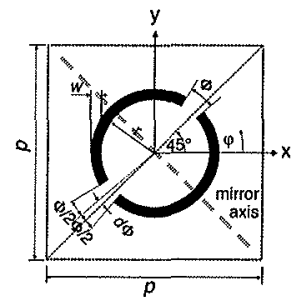

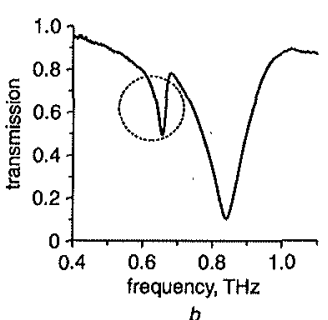

Fig. 1 Lateral unit cell structure and transmission characteristic of FSS $a$ Lateral unit cell structure of FSS

Resonators dimensioned with $w=10 \mu \mathrm{m}, \Phi=22^{\circ}, \mathrm{d} \Phi=5^{\circ}, p=197 \mu \mathrm{m}, r=$ $49 \mu \mathrm{m}$

$b$ Transmission characteristic of FSS

Experimental setup: The terahertz transmission through the FSS was investigated by time-domain terahertz spectroscopy using a system based on high-speed ASOPS [11]. This time-domain terahertz spectrometer provides a unique combination of long time delay (1 ns) and high dynamic range without a mechanical delay line. The electric field of the terahertz wave is detected in an electro-optic crystal. The Fourier transform of the electric field detected in the time domain yields a $1 \mathrm{GHz}$ frequency resolution with a $44 \mathrm{~dB}$ dynamic range in $1 \mathrm{~s}$ of data acquisition. The polarisation of the terahertz radiation is chosen perpendicular to the mirror axis of the FSS as depicted in Fig. $1 a$ in order to excite the highest extinction fundamental resonance of the FSS.

Results: The transmission through the FSS is shown in Fig. 1b. The resonator array is equipped with an element perturbation such that every arc pair provides two slightly different resonant frequencies. Consequentially, the transmission spectrum exhibits the expected devolution with a local transmission maximum in between a narrow and a broad absorption line generated by modal interaction as described by Larson and Munk [12]. The local transmission maximum appears when the array self-reactance $X_{A}$ becomes equal to the sum mutual reactance $X_{M}$. In this case, the currents are excited in anti-phase. The scattered electromagnetic fields interfere destructively, so the coupling to free space is strongly reduced. Fedotov et al. denote this as a trapped mode' [13]. For sensing applications this mode is extremely attractive, since it offers strong field localisation and temporal remaining energy storage at the resonator surface where thin films of sample material are to be detected. To evaluate the frequency position of the modal-interaction resonance, a function based on two modified sigmoid functions is fitted to the first minimum (see circle in Fig. $1 b$ and inset of Fig. 2).

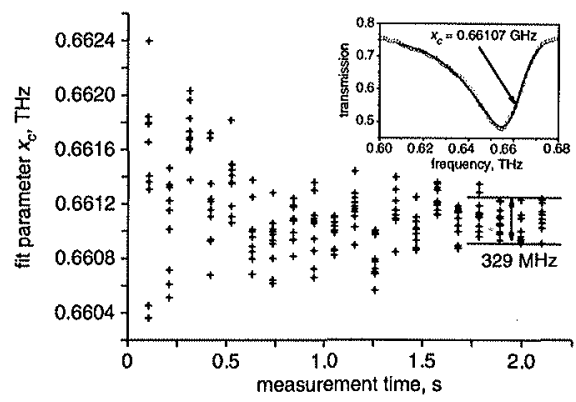

Fig. 2 Fit parameter $x_{c}$ plotted against measurement time Inset: Experimental data (circles) and fit function (solid line)

The function contains five fit parameters

$f(x)=y_{0}$

$$
+A *\left(\frac{1}{1+\exp \left(-\left(x-x_{c}\right) / w_{1}\right)}\right) *\left(1-\frac{1}{1+\exp \left(-\left(x-x_{c}\right) / w_{2}\right)}\right)
$$

$y_{0}$ is the offset, $A$ the amplitude, $w_{1}$ the falling edge, $w_{2}$ the rising edge and $x_{c}$ describes the position of the resonance (see inset Fig. 2). Since the fit function is transcendent, the function and its derivatives cannot be analytically solved for $x_{c}$. However, it can be shown that $x_{c}$ must be located between the minimum and the second inflection point. The fit ranges from $0.608-0.673 \mathrm{THz}$ and consists of 55 data points. To test the relation between precision and measurement time, we performed ten individual measurements for different acquisition times ranging from $0.1-2.1 \mathrm{~s}$. In total, 200 spectra were fitted. For every fit the parameter $x_{c}$ converges against a fixed value without adjusting the other fit parameters. Fig. 2 shows the fit parameter $x_{c}$ against measurement time. The scatter of the $x_{c}$ values decreases with measurement time. For measurement times greater than $1.9 \mathrm{~s}$ the scatter range is below $330 \mathrm{MHz}$. The statistical error of $x_{c}$ for an individual measurement is about $180 \mathrm{MHz}$. The combination of the scattering of $x_{c}$ and the statistical error leads to a mean value of $x_{c}=0.66113 \mathrm{THz} \pm 325 \mathrm{MHz}$ within a read-out time of $2.1 \mathrm{~s}$.

One factor affecting the precision of the resonance determination is the accuracy of the spectrometer time-axis calibration. Tests have shown that our high-speed ASOPS spectrometer has a relative error well below $10^{-4}[11]$, resulting in a frequency error of less than $66 \mathrm{MHz}$ for the above measurement. This error, however, is common to all measurements and does not affect the repeatability of a measurement or the mere determination of a resonance shift. A second important parameter influencing the resonance evaluation is the dynamic range of the obtained Fourier spectra. An increasing dynamic range reduces the error of the fitting procedure and can enhance the obtainable precision of the resonance determination to values well below the given $1 \mathrm{GHz}$ instrument resolution. Both criteria, precise time-axis calibration and high dynamic range, can also be fulfilled with a conventional terahertz 
time-domain spectrometer operating with a mechanical delay stage [10]. However, the key advantage of high-speed ASOPS in contrast to conventional systems is that it is capable of directly measuring at the shot noise limit without further noise suppression measures (e.g. balanced detection, lock-in detection). This is because the $10 \mathrm{kHz}$ scan frequency is well above typical Fourier components contained in the technical noise affecting the experiment. In addition, no time is required for acceleration and deceleration of a translation stage between the acquisitions of two data points. Both above advantages are the basis for a significantly enhanced data acquisition speed.

Conclusions: We have shown that a terahertz spectrometer based on high-speed ASOPS can determine a characteristic frequency of a $\mathrm{THz}-$ FSS array with a relative uncertainty of $1 \times 10^{-3}$ within a measurement time of only 2 seconds. This result is made possible through a unique combination of 1 ns scan range, i.e. $1 \mathrm{GHz}$ instrument resolution, with a rapid high dynamic range data acquisition. The method may be highly beneficial for fast sensing of small amounts of bio-materials on a THz-FSS

Acknowledgments: This research is supported by the Landesstiftung Baden-Württemberg, Financial support of the Center for Applied Photonics (CAP) is gratefully acknowledged.

G. Klatt, T. Dekorsy and A. Bartels (Department of Physics and Center for Applied Photonics, University of Konstanz, Konstanz D-78457, Germany)

E-mail: Gregor.Klatt@uni-konstanz.de

M. Nagel (Institute of Semiconductor Electronics, RWTH Aachen University, Aachen 52074, Germany)

\section{References}

1 Munk, B.A.: 'Frequency selective surfaces: theory and design' (WileyInterscience, 2000)

2 Debus, C., and Bolivar, P.H.: 'Frequency selective surfaces for high sensitivity terahertz sensing', Appl. Phys. Lett., 2007, 91, p. 184102

3 Nagel, M., et al.: 'A functionalized THz sensor for marker-free DNA analysis', Phys. Med. Biol, 2003, 48, pp. 3625-3636

4 Singh, R., et al.: 'Optically thin terahertz metamaterials', Opt. Express, 2008,16 , pp. $6537-6543$

5 Yoshida, H., et al.: 'Terahertz sensing method for protein detection using a thin metallic mesh', Appl. Phys. Lett., 2007, 91, p. 253901

6 O'Hara, J.F., et al:: 'Thin-film sensing with planar terahertz metamaterials: sensitivity and limitations', Opt. Express, 2008, 16, pp. $1786-1795$

7 Al-Naib, I.A.I., et al:: 'Thin-film sensing with planar asymmetric metamaterial resonators', Appl. Phys. Lett., 2008, 93, p. 083507

8 Brucherseifer, M., et al.: 'Label-free probing of the binding state of DNA by time-domain terahertz sensing', Appl. Phys. Lett., 2000, 77, pp. 4049-4051

9 Globus, T., et al.: 'Terahertz Fourier transform characterization of biological materials in a liquid phase', J. Phys. D, Appl. Phys., 2006, 39 , pp. $3405-3413$

10 Exter, M.v., et al.: 'Terahertz time-domain spectroscopy of water vapor', Opt. Lett., 1989, 14, pp. 1128-1130

11 Bartels, A., et al:: 'Ultrafast time-domain spectroscopy based on highspeed asynchronous optical sampling', Rev. Sci. Instrun., 2007, 78, p. 035107

12 Larson, C., and Munk, B.: 'The broad-band scattering response of periodic arrays', IEEE Trans. Antennas Propag., 1983, 31, pp. 261-267

13 Fedotov, V.A., et al:: 'Sharp trapped-mode resonances in planar metamaterials with a broken structural symmetry', Phys. Rev. Lett., 2007,99 , p. 147401 